\title{
A novel over-sampling method and its application to miRNA prediction
}

\author{
Xuan Tho Dang ${ }^{*}$, Osamu Hirose ${ }^{2}$, Thammakorn Saethang ${ }^{1}$, Vu Anh Tran ${ }^{1}$, Lan Anh T. Nguyen ${ }^{1}$, \\ Tu Kien T. Le ${ }^{1}$, Mamoru Kubo ${ }^{2}$, Yoichi Yamada ${ }^{2}$, Kenji Satou ${ }^{2}$ \\ ${ }^{1}$ Graduate School of Natural Science and Technology, Kanazawa University, Kanazawa, Japan \\ ${ }^{2}$ Institute of Science and Engineering, Kanazawa University, Kanazawa, Japan \\ Email: "thodx@hnue.edu.vn
}

Received 15 December 2012; revised 14 January 2013; accepted 20 January 2013

\begin{abstract}
MicroRNAs (miRNAs) are short ( 22 nt) non-coding RNAs that play an indispensable role in gene regulation of many biological processes. Most of current computational, comparative, and non-comparative methods commonly classify human precursor microRNA (pre-miRNA) hairpins from both genome pseudo hairpins and other non-coding RNAs (ncRNAs). Although there were a few approaches achieving promising results in applying class imbalance learning methods, this issue has still not solved completely and successfully yet by the existing methods because of imbalanced class distribution in the datasets. For example, SMOTE is a famous and general over-sampling method addressing this problem, however in some cases it cannot improve or sometimes reduces classification performance. Therefore, we developed a novel over-sampling method named incre-mentalSMOTE to distinguish human pre-miRNA hairpins from both genome pseudo hairpins and other ncRNAs. Experimental results on pre-miRNA datasets from Batuwita et al. showed that our method achieved better Sensitivity and G-mean than the control (no oversampling), SMOTE, and several successsors of modified SMOTE including safe-level-SMOTE and border-line-SMOTE. In addition, we also applied the novel method to five imbalanced benchmark datasets from UCI Machine Learning Repository and achieved improvements in Sensitivity and G-mean. These results suggest that our method outperforms SMOTE and several successors of it in various biomedical classification problems including miRNA classification.
\end{abstract}

Keywords: Imbalanced Dataset; Over-Sampling; SMOTE; miRNA Classification

"Corresponding author.

\section{INTRODUCTION}

MicroRNAs (miRNAs) are short ( 22nt) non-coding RNAs (ncRNAs) that play an indispensable role in gene regulation of many biological processes. The tiny miRNAs can target numerous mRNAs to induce mRNA degradation or translational repression or both, they could regulate $20 \%-30 \%$ of human genes [1]. The miRNAs are transcribed as long primary miRNAs (pri-miRNAs) which are processed into 60 - $70 \mathrm{nt}$ precursor miRNAs (premiRNAs) by Drosha-DGCR8. The pre-miRNA is transferred from Nucleus to Cytoplasm by Exp5-RanGTP and then split by Dicer-TRBP into miRNA duplex ( 22 nt) [1].

The first miRNAs were characterized in early 1990s [2] but research on miRNAs has not revealed multiple roles in gene regulation (transcript degradation, translational suppression or transcriptional and translational activation) yet. Until the early 2000s, miRNAs were recognized as essential components in most biological processes [3-6]. Subsequently, miRNAs have become a hot topic and a large number of miRNAs, particularly 21,264 different miRNAs have been identified in various species so far according to the release 19 of miRBase [7]. However, the identification of miRNAs from a genome by existing experiment techniques is so difficult, expensive, and requires a large amount of time. Therefore, computational methods with two main approaches based on comparative and non-comparative methods play important role to detect new miRNAs. The rationale idea of the first approach-comparative methods is that miRNA genes are conserved in closely related genomes in hairpin seconddary structures. Several comparative methods are presented such as RNAmicro [8], MiRscan [9], miRseeker [10], MIRcheck [11], and MiRFinder [12]. These conservation-dependent comparative methods are successful to predict hundreds of miRNAs with high sensitivity in closely related species. However, they are unable to identify novel miRNAs without close homologies due to lack of current data or unreliability of alignment algorithms [13], especially due to possibly rapid evolution of 
miRNAs. Particularly, the report from Berezikov et al. [14] has emphasized that non-conserved miRNAs in human genome missed by comparative methods are relatively large and even have not still been recognized yet.

Meanwhile, the second approach-non-comparative methods are promising for recognizing additional miRNAs which are non-conserved miRNAs. The main idea of these methods is based on hairpin secondary structures of pre-miRNAs. Sewer et al. [15] used clustering approach to predict novel miRNAs in the same cluster with known miRNAs. Xue et al. [16] proposed the classification of real and pseudo pre-miRNA hairpins based on 32 features of structure-sequence triplet. Clote et al. [17] and Hertel et al. [8] also identified that hairpin secondary structures are popular in many types of ncRNAs and a huge number of pseudo hairpins and hairpin structures can be found among secondary structures of other ncRNAs. In addition, machine learning approaches including random forest prediction model [18, 19]; hybrid of genetic algorithm and support vector machine (GA-SVM) [20]; feature selection strategies based on SVM and boosting method [21]; hidden Markov model (HMM) [22] have also been used.

Therefore, both genome pseudo hairpins, other ncRNAs and machine learning approaches should be applied. In addition, miRNA prediction problem should be also considered as imbalance class distribution. Batuwita et al. [23] proposed an effective classifier system, namely microPred which used a complete pseudo hairpin dataset and ncRNAs. In their research, they focused on handling with class imbalance problem in datasets where samples from the majority class $(9248=8494$ pseudo hairpins +754 other ncRNAs) significantly outnumber the minority class (691 pre-miRNAs). Xiao et al. [24] presented several network parameters based on two-dimensional network of pre-miRNA secondary structure such as bracketed, tree, dual graph, etc. Their dataset contained 3928 positive samples (animal pre-miRNAs) and 8897 negative samples (8487 pseudo hairpins and 410 ncRNAs). Therefore, this dataset is suffered from imbalance problem, that is, the negative dataset outnumbers the positive dataset. The main problem of class imbalance distribution is that normal learners are often biased to the majority class, leading to good classification for the majority class samples while misclassification for many minority class ones. In order to solve these class imbalance learning problems, some solutions have been developed, including two main types: the external methods at data processing level and the internal methods at algorithm level. One of remarkable solutions is SMOTE which is a famous and general over-sampling method addressing this problem. For example, experimental results of Batuwita et al. [23] suggested that the best classifier has been developed by applying the SMOTE method. However, there are still some drawbacks of SMOTE, particularly in some cases it cannot improve or sometimes reduces classification performance. Therefore, in our research we developed a novel over-sampling method to achieve better classification performance than both of the control method (no over-sampling) and SMOTE in the classification of pre-miRNAs for human miRNA gene prediction. Moreover, in order to demonstrate the applicability of our methods, we also compare our methods with several successors of modified SMOTE including safe-level-SMOTE [25] and borderline-SMOTE [26].

The structure of this paper is organized as follows: Section 2 gives a brief introduction to SMOTE and some related works, then shows its drawback; Section 3 introduces three variations of our novel method, incrementalSMOTE, which is improved from SMOTE; Section 4 analyses the experiments and compares our novel method with the control, SMOTE, safe-level-SMOTE, and borderline-SMOTE methods. Finally, conclusions are described in Section 5.

\section{METHOD}

\subsection{SMOTE}

Chawla et al. developed a minority over-sampling technique called SMOTE [27] in which the minority class samples are over-sampled by creating synthetic samples rather than by over-sampling with replacement. SMOTE provided a new approach to over-sampling and introduced a bias towards the minority class. The results in [27] showed that this approach could improve the performance of classifiers for the minority class.

In a less application-specific manner, synthetic samples are generated by operating in "feature space" rather than "data space". The minority class is over-sampled by synthesizing new samples along the line joining the minority samples and their nearest neighbors. Depending on the requirement of over-sampling amount, nearest neighbors are selected by chance. Synthetic samples are generated in the following way: firstly, compute the difference of feature vector between each minority class sample and its randomly selected nearest neighbor. Then, multiply this difference by a random number between 0 and 1 , and finally add it to the feature vector of the minority sample. In this way, the synthetic minority sample is generated along the line segment between two specific features.

This approach is effective in forcing the decision region of the minority class to become more general as shown in Figure 1. Figure 1(a) presents a typical case of imbalanced data where samples from the majority class greatly outnumber the minority class. As a result, the majority class samples are well-classified whereas many 
samples from the minority class are easy to be misclassified. Therefore, imbalanced dataset problem requires a new and more adaptive method such as SMOTE. Figure 1(b) describes synthetic samples generated by SMOTE to achieve more balanced distribution so that the classifier recognizes all samples correctly.

A variation of SMOTE, namely borderline-SMOTE is proposed by Han et al. [26] as the improvement of SMOTE. The authors analyzed most of the classification algorithms and attempt to learn the borderline of each class as exactly as possible in the training process. Those samples far from the borderline may make a little contribution to classification. Therefore, their method was based on the same over-sampling technique to SMOTE, but the difference is it only over-sampled the borderline samples of minority class instead of over-sampling all samples of the class as in SMOTE.

Another modification of SMOTE, safe-level-SMOTE is also presented by Bunkhumpornpat et al. [25]. Instead of randomly synthesizing the minority samples along the line joining a minority sample and its selected nearest neighbours, this method ignored nearby majority samples. The safe-level-SMOTE method carefully generated synthetic samples along the same line with different weight level, called safe level. The safe level was computed by using nearest neighbour minority samples.

Although SMOTE and several successors of modified SMOTE including borderline-SMOTE and safe-levelSMOTE are famous and general over-sampling methods addressing the imbalanced class distribution problems, in some cases they cannot improve or sometimes reduce classification accuracy.

\subsection{Drawback of SMOTE}

As discussed above, one particular synthetic sample is generated by using SMOTE as shown in Figure 2(a). Blue sample $\mathrm{x}$ is a synthetic sample generated along the line joining a minority class sample s and its randomly selected nearest neighbor d. Generally, the set of syn- thetic samples are generated between two sets of minority class samples named Source (S) and Destination (D). Figure 2(b) describes step by step the generation of synthetic samples by using SMOTE method: at the first step, a set of synthetic samples (X1) is generated, and at the next step, another new set (X2) is generated; the process is repeated and the sets of generated synthetic samples can be different in each step.

In Figure 2(b), we could realize the drawbacks of SMOTE method: $\mathrm{S}$ and $\mathrm{D}$ do not change in the process of generating synthetic samples; and synthetic minority class samples will not be paid any attention after they are generated. Therefore, in order to address these drawbacks and improve classification accuracy of the SMOTE method, we focus on how to utilize generated synthetic samples as members of Source and Destination sets for further generation. This idea will be presented in next section, a novel method namely incremental- SMOTE.

Moreover, although $\mathrm{X}$ is generated by $\mathrm{S}$ and $\mathrm{D}, \mathrm{S}$ is the decisive factor in generating $\mathrm{X}$. In contrast, $\mathrm{D}$ is only a randomly selected nearest neighbor to be used in combination with $\mathrm{S}$ in this generation. Therefore, the change of $\mathrm{S}$ will lead to the change of $\mathrm{X}$ as shown below in incremental-SMOTE2 and incremental-SMOTE3.

Based on the analysis, we present three new methods, incremental-SMOTE1, incremental-SMOTE2, and incremental-SMOTE3.

\subsection{Incremental-SMOTE1}

The idea of incremental-SMOTE1 is simple: the Destination set is expanded incrementally while keeping the Source set to be unchanged in all steps. Figure 3 illustrates more details about this idea. In step 1, using SMOTE, a set of synthetic samples X1 is generated from two sets-Source (S) and Destination (D) sets as mentioned above. In step 2, the Destination set is expanded by merging X1 into it. Similarly in step 3, the Destination set is expanded again by merging $\mathrm{X} 2$ into it. The process is repeated in further steps.

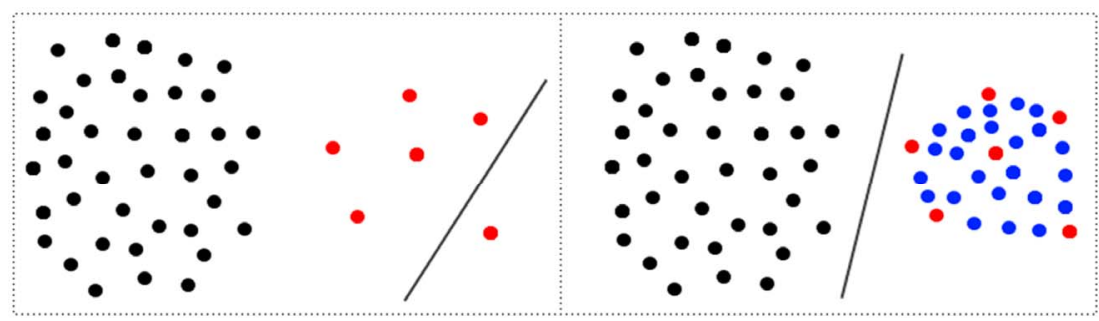

$\bullet$ majority class samples • minority class samples • synthetic sample

(a)

(b)

Figure 1. Advantages of SMOTE. Black, red, and blue dots indicate majority class samples, minority class samples, and synthetic minority class samples, respectively. The discrimination hyperplane is the brown line. (a) The original dataset with an erroneous classifier biased by the imbalanced dataset; (b) Synthesize some new minority class samples by applying SMOTE with a perfect classifier. 


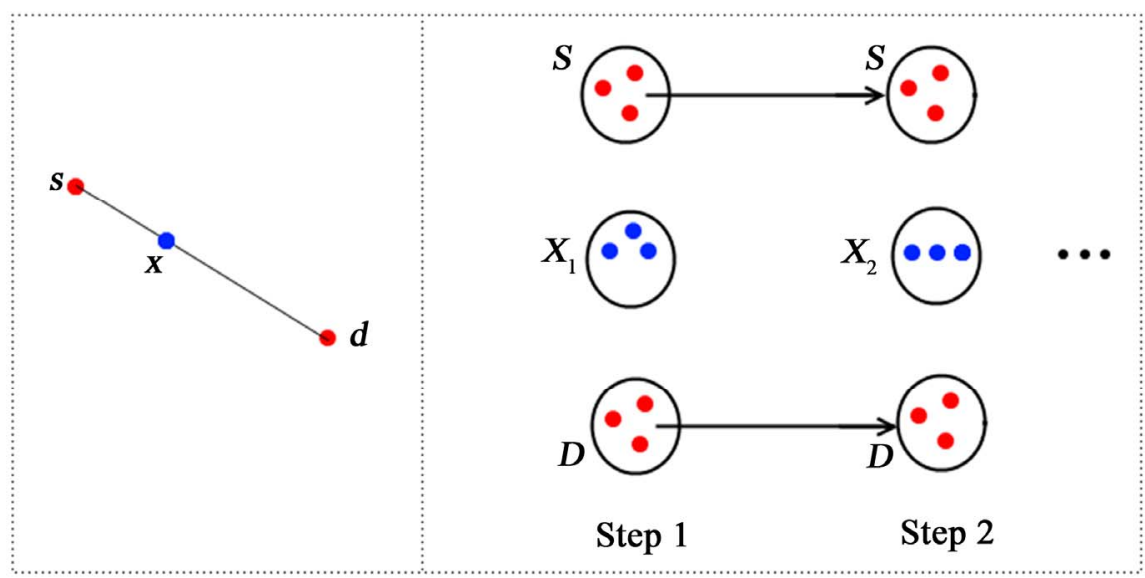

(a)

(b)

Figure 2. Synthetic samples generated normally by SMOTE. Both red and blue dots indicate minority class samples. The latter indicate synthetic minority class samples generated by using SMOTE. (a) Particularly, one synthetic minority class sample is generated along the line of two minority class samples by using SMOTE; (b) Generally, a set of synthetic samples is generated from two sets named Source (S) and Destination (D).
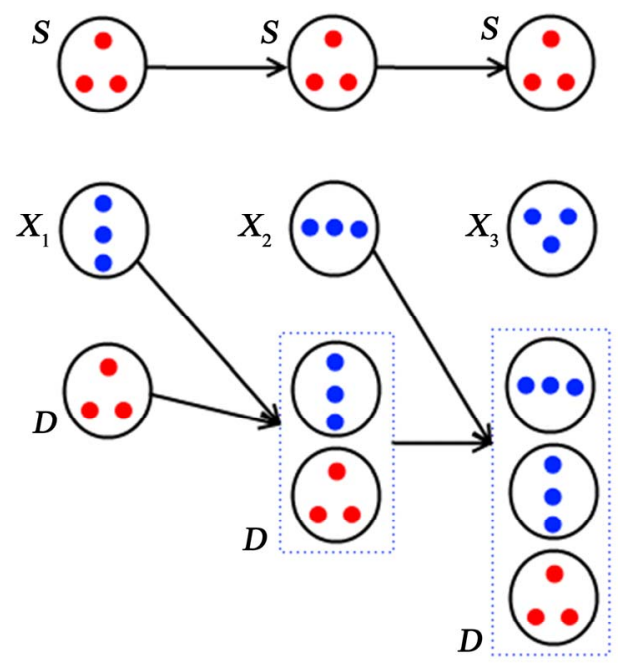

Step $1 \quad$ Step $2 \quad$ Step 3

Figure 3. The idea of incremental-SMOTE1.

Pseudo-code of incremental-SMOTE1 is as follows: Algorithm incremental-SMOTE1 (T, N).

Input: The number of minority class samples $\mathrm{T}$; Amount of incremental-SMOTE1 N (\%);

Output: $(\mathrm{N} \times \mathrm{T} / 100)$ synthetic minority class samples

$0)$ Initialize and assign two new sets with the same size as the number of minority class samples.

Source $=\mathrm{T}$; Destination $=\mathrm{T}$;

1) Generate the new set $X$ of $T$ synthetic samples by using SMOTE.

$\mathrm{X}=$ new synthetic samples generated at this step;

2) Merge $X$ into the Destination set.

Destination $=$ Destination $+\mathrm{X}$;

3) Repeat from Step 1 N/100 times.

\subsection{Incremental-SMOTE2}

Incremental-SMOTE2 is based on a reversal idea: the Destination set now is kept to be the same in every step, and the Source set is expanded incrementally. It is shown clearly in Figure 4.

Pseudo-code of incremental-SMOTE2 is as follows:

Algorithm incremental-SMOTE2 (T, N).

Input: The number of minority class samples $\mathrm{T}$; Amount of incremental-SMOTE2 N (\%);

Output: $(\mathrm{N} \times \mathrm{T} / 100)$ synthetic minority class samples

0 . Initialize and assign two new sets with the same size as the number of minority class samples.

Source $=\mathrm{T}$; Destination $=\mathrm{T}$;

1) Generate the new set $X$ of $T$ synthetic samples by using SMOTE.

$\mathrm{X}=$ new synthetic samples generated at this step;

2) Merge $X$ into the Source set.

Source $=$ Source $+\mathrm{X}$;

3) Repeat from Step 1 until $(\mathrm{N} \times \mathrm{T} / 100)$ synthetic minority class samples are generated.

\subsection{Incremental-SMOTE3}

The idea of incremental-SMOTE3 is the combination of incremental-SMOTE1 and incremental-SMOTE2: both Source and Destination sets are expanded. Figure 5 shows more details for this idea.

Pseudo-code of incremental-SMOTE3 is as follows:

Algorithm incremental-SMOTE3 (T, N).

Input: The number of minority class samples $\mathrm{T}$; Amount of SMOTE N (\%);

Output: $(\mathrm{N} * \mathrm{~T} / 100)$ synthetic minority class samples

$0)$ Initialize and assign two new sets with the same size as the number of minority class samples. 


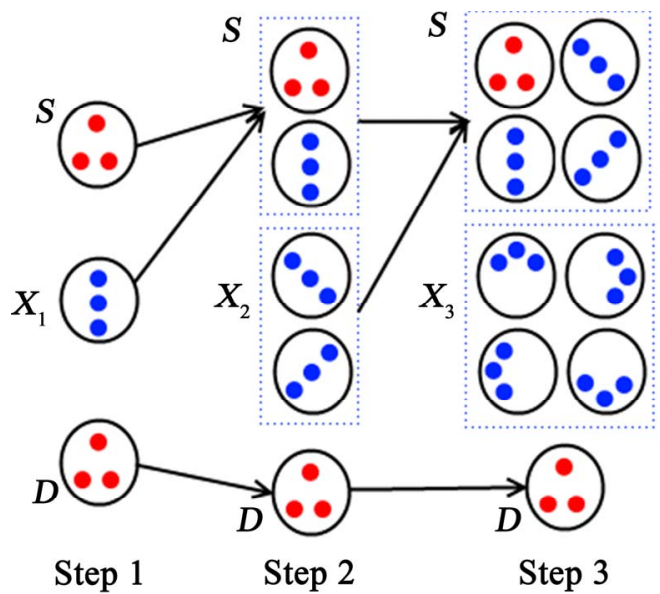

Figure 4. The idea of incremental-SMOTE2.

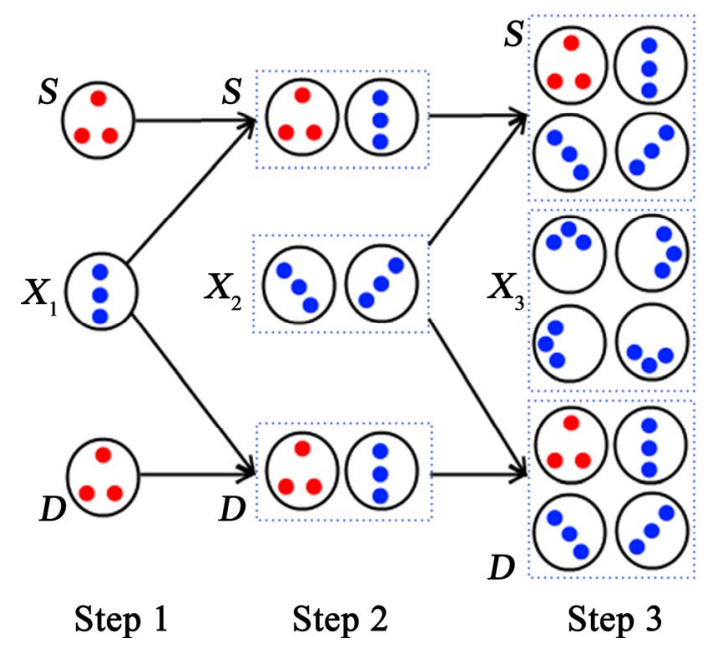

Figure 5. Thes idea of incremental-SMOTE3.

Source $=\mathrm{T}$; Destination $=\mathrm{T}$;

1) Generate the new set $X$ of $T$ synthetic samples by using SMOTE.

$\mathrm{X}=$ new synthetic samples generated at this step;

2) Merge $X$ into the Source and Destination sets.

Source $=$ Source $+\mathrm{X}$;

Destination $=$ Destination $+\mathrm{X}$;

3) Repeat from Step 1 until $(N \times T / 100)$ synthetic minority class samples are generated.

\subsection{Classifier}

For binary class classification, Support Vector Machine (SVM) is widely used to build a classifier discriminating the classes [28]. SVM is based on simple ideas originated in statistical learning theory [29] which has high generalization capability, optimizes global classification solution and could be successfully applied in bioinformatics. In addition, in order to more generally evaluate the performance of our method, two different classification methods other than SVM were used, namely k-
Nearest Neighbour (k-NN) and Random Forest (RF).

Implementation of SVM, k-NN, and RF in kernlab [30], class [31], and random Forest [32]package available at the Comprehensive R Archive Network (CRAN) was used, respectively. In our research, we used Radial Basis kernel (Gaussian kernel) of kernlab for SVM. Kernlab is an extensible package for kernel-based machine learning methods in $\mathrm{R}$ and includes various kernels such as Linear kernel, Radial Basis kernel (Gaussian kernel), Polynomial kernel, etc. Moreover, all hyperparameters for k-NN and RF, as well as other hyper-parameters for SVM such as cost, class weights, etc. were also set to be default values.

\subsection{Evaluation Measures}

A confusion matrix of binary class classification is shown in Table 1. In the field of binary classification in imbalanced data, most of the studies consider the class label of the minority class as positive. Thus, the class labels positive and negative are given to the samples in minority and majority classes, respectively. In Table 1, the first column presents the actual class label of the samples, and the first row is their predicted outcome class label. TP and TN denote the number of positive and negative samples that are classified correctly, while FN and FP denote the number of misclassified positive and negative samples, respectively.

If the dataset is extremely imbalanced, for example, with an imbalance ratio of 99 to 1 , even when the classifier classifies all the samples as negative, the accuracy of classification is still high up to $99 \%$. As a result, accuracy is not used to evaluate the performance of classifier for imbalance datasets, and more reasonable evaluation metrics should be presented [33,34].

In medical science, bioinformatics, and machine learning communities $[23,24,33,34]$, the sensitivity (SE) and the specificity (SP) are two metrics used to evaluate the performance of classifiers. Sensitivity measures the proportion of actual positives which are correctly identified as such, while specificity can be defined as the proportion of negatives which are correctly identified. Kubat et al. [35] proposed the Geometric mean metric defined as follows.

$$
\mathrm{G}-\mathrm{mean}=\sqrt{\mathrm{SE} \times \mathrm{SP}}
$$

There are many researches applying this metric for evaluating classifiers as commonly used in imbalanced

Table 1. A confusion matrix for binary class classification.

\begin{tabular}{ccc}
\hline & Predicted Positive & Predicted Negative \\
\hline Observed Positive & TP & FN \\
Observed Negative & FP & TN \\
\hline
\end{tabular}


class distribution [23,34-37]. Therefore, we use this metric to measure the performances of the classifiers in our research.

\section{RESULTS}

\section{1. miRNA Dataset}

The miRNA datasets selected in this research were downloaded from the website of microPred classifier system [23] and miRNA network-level [24]. MicroPred consists of three kinds of non-redundant human sequences: 691 pre-miRNAs, 8494 pseudo hairpins, and 754 other ncRNAs (9248 hairpins). The first type is positive, and the others are negative. Meanwhile, miRNA network-level dataset contains 3928 positive samples (animal pre-miRNAs) and 8897 negative samples (8487 pseudo hairpins and 410 ncRNAs). Class imbalance ratio of the positive to negative dataset of microPred and miRNA network-level was $1: 13$ and $1: 2$, respectively. It means that these datasets have imbalanced class distribution with majority class samples outnumbering minority ones. In microPred dataset, 48 features were used to represent each sample while there were only 21 features used in miRNA network-level dataset. This was shown clearly by Xiao et al. [24] who presented 24 network features but three of them (Girth, M_coreness, and Transitivity) were meaningless because all samples are the same value in both classes. Subsequently, the rest 21 features were meaningful and thus used in miRNA network-level dataset.

\subsection{Classification Imbalance Learning Results}

The experiments were executed to compare five methods: control method (no over-sampling), SMOTE, safe-levelSMOTE, borderline-SMOTE, and incremental-SMOTE. SVM, k-NN, and RF were used as the classifiers. The classification performance of the methods was estimated by 10 -fold cross-validation. For each test, nine-tenth of the complete dataset was used as a training set. Then, in case of SMOTE, safe-level-SMOTE, borderline-SMOTE, and incremental-SMOTE, minority samples in the training set over-sampled with the value of $\mathrm{k}$ is set to 5 like as SMOTE. After the training by an SVM, k-NN, or RF model using the (possibly over-sampled) training set, the model was tested against the remaining one-tenth of the dataset (i.e. test set). This process was repeated for all 10 -fold with different combination of training and test sets. The values for the criteria of performance, sensitivity, specificity, and G-mean were calculated by averaging 20 independent runs of 10-fold cross-validation and summarized in Table 2. Furthermore, two-sample t-test with equal variance was conducted to assess whether the averages of G-mean by different methods are significantly different.

Table 2. Classification performance for microPred and miRNA network-level datasets expressed in percent.

\begin{tabular}{|c|c|c|c|c|c|c|c|c|c|c|}
\hline \multirow{2}{*}{ Dataset } & \multirow{2}{*}{ Method } & \multicolumn{3}{|c|}{ SVM } & \multicolumn{3}{|c|}{$\mathrm{k}-\mathrm{NN}$} & \multicolumn{3}{|c|}{$\mathrm{RF}$} \\
\hline & & $\mathrm{SE}$ & SP & G-mean & $\mathrm{SE}$ & SP & G-mean & SE & SP & G-mean \\
\hline \multirow{8}{*}{ microPred } & No over-sampling & 97.82 & 99.98 & 98.89 & 58.54 & 99.82 & 76.44 & 99.46 & 99.97 & 99.72 \\
\hline & SMOTE & 98.68 & 99.96 & 99.32 & 81.40 & 98.06 & 89.34 & 99.70 & 99.97 & 99.84 \\
\hline & increSMOTE1 & 99.10 & 99.95 & 99.52 & 84.74 & 97.26 & 90.78 & 99.78 & 99.97 & 99.88 \\
\hline & increSMOTE2 & 98.94 & 99.95 & 99.44 & 90.98 & 94.52 & 92.74 & 99.80 & 99.97 & 99.88 \\
\hline & increSMOTE3 & 98.93 & 99.95 & 99.44 & 89.49 & 94.66 & 92.04 & 99.79 & 99.97 & 99.88 \\
\hline & Safe level-SMOTE & 98.72 & 99.96 & 99.34 & 81.27 & 97.53 & 89.03 & 99.72 & 99.97 & 99.84 \\
\hline & Borderline-SMOTE1 & 98.65 & 99.96 & 99.30 & 79.39 & 97.73 & 88.08 & 99.64 & 99.97 & 99.80 \\
\hline & Borderline-SMOTE2 & 98.80 & 99.93 & 99.36 & 83.93 & 96.58 & 90.03 & 99.97 & 99.94 & 99.96 \\
\hline \multirow{8}{*}{$\begin{array}{l}\text { miRNA } \\
\text { network } \\
\text { level }\end{array}$} & No over-sampling & 80.43 & 96.51 & 88.10 & 79.85 & 94.92 & 87.06 & 82.58 & 95.92 & 89.00 \\
\hline & SMOTE & 88.08 & 91.32 & 89.69 & 84.77 & 90.23 & 87.46 & 87.28 & 91.82 & 89.52 \\
\hline & increSMOTE1 & 88.57 & 90.99 & 89.77 & 84.69 & 90.74 & 87.67 & 86.24 & 93.02 & 89.57 \\
\hline & increSMOTE2 & 89.62 & 89.31 & 89.47 & 87.58 & 85.71 & 86.64 & 87.01 & 92.12 & 89.53 \\
\hline & increSMOTE3 & 89.67 & 89.24 & 89.45 & 87.47 & 85.27 & 86.36 & 86.38 & 92.78 & 89.52 \\
\hline & Safelevel-SMOTE & 88.22 & 91.20 & 89.70 & 84.97 & 89.84 & 87.37 & 85.66 & 93.51 & 89.50 \\
\hline & Borderline-SMOTE1 & 86.58 & 93.14 & 89.80 & 83.35 & 91.25 & 87.21 & 85.47 & 93.59 & 89.44 \\
\hline & Borderline-SMOTE2 & 86.63 & 93.01 & 89.76 & 86.09 & 85.50 & 85.80 & 85.09 & 93.92 & 89.39 \\
\hline
\end{tabular}


Table 3. The description of the imbalanced datasets from UCI.

\begin{tabular}{lccc}
\hline \multicolumn{1}{c}{ Name } & Examples & Attributes & Imbalance ratio \\
\hline Breast-w & 683 & 10 & $1: 1.90$ \\
Haberman & 306 & 3 & $1: 2.78$ \\
Blood & 748 & 4 & $1: 3.20$ \\
Breast-p & 198 & 32 & $1: 3.21$ \\
Yeast & 1484 & 8 & $1: 28.10$ \\
Ionosphere & 351 & 34 & $1: 1.79$ \\
Glass & 214 & 9 & $1: 6.38$ \\
Satimage & 6435 & 36 & $1: 9.28$ \\
\hline
\end{tabular}

Experimental results on the microPred dataset showed that our method achieved better G-mean than control method, SMOTE, safe-level-SMOTE, and borderlineSMOTE on two of three classifiers. For example, with using SVM, although the sensitivity and G-mean increased for control method $(97.82 \%$ and $98.89 \%)$, SMOTE $(98.68 \%$ and $99.32 \%)$, safe-level-SMOTE (98.72\% and $99.34 \%)$, borderline-SMOTE1 $(98.65 \%$ and $99.30 \%)$, and borderline-SMOTE2 (98.80\% and $99.36 \%)$, they also increased by $(99.10 \%$ and $99.52 \%),(98.94 \%$ and $99.44 \%$ ), and $(98.93 \%$ and $99.44 \%)$ for incrementalSMOTE1, incremental-SMOTE2, and incrementalSMOTE3, respectively. However, it is different in the criterion of the specificity: in comparison with control method $(99.98 \%)$, the specificity was decreased by $0.02 \%$ for SMOTE, safe-level-SMOTE, and borderlineSMOTE1 (99.96\%, 99.96\% and 99.96\%); $0.03 \%$ for incremental-SMOTE1, incremental-SMOTE2, and incremental-SMOTE3 (99.95\%, 99.95\%, 99.95\%, and 99.95\%); and $0.05 \%$ for borderline-SMOTE2 $(99.93 \%)$.

The assessment by t-test on the microPred dataset suggested that SMOTE, safe-level-SMOTE, borderlineSMOTE1, and borderline-SMOTE2 significantly outperforms the control method (with p-value $2.2 \mathrm{E}-16$, $2.2 \mathrm{E}-16,4.7 \mathrm{E}-15$, and $2.2 \mathrm{E}-16$, respectively) and this is also similar with incremental-SMOTE1, incrementalSMOTE2, and incremental-SMOTE3 in comparison with the control method (with p-values $7.24 \mathrm{E}-14,4.5 \mathrm{E}-13$, and $6.2 \mathrm{E}-10$, respectively). Furthermore, it is easily recognized that three methods used in our research also remarkably outperform SMOTE, safelevel-SMOTE, borderline-SMOTE1, and borderline-SMOTE2 with p-values $(5.4 \mathrm{E}-5,3.7 \mathrm{E}-3$, and $2.1 \mathrm{E}-2) ;(1.9 \mathrm{E}-4,1.2 \mathrm{E}-2$, and $4.0 \mathrm{E}-2) ;(3.4 \mathrm{E}-5,2.4 \mathrm{E}-3$, and $1.3 \mathrm{E}-2)$; and $(5.6 \mathrm{E}-4$, $3.2 \mathrm{E}-2$, and $8.0 \mathrm{E}-2)$, respectively.

In addition, the experimental results and the assessment by t-test on the miRNA network-level dataset also suggested that our method significantly achieved better G-mean than control method, SMOTE, and safe-levelSMOTE by using all three classifiers, and significantly outperformed borderline-SMOTE on two of three classi- fiers (for more details, see Tables 2 and 5).

\subsection{Benchmark Datasets}

To demonstrate the applicability of our methods, we also performed experiments using eight real-world imbalanced benchmark datasets from UCI Machine Learning Repository [38]: Radar data (ionosphere), Breast Cancer Wisconsin (breast-w), Haberman's Survival (haberman), Blood Transfusion Service Center (blood), Wisconsin Prognostic Breast Cancer (breast-p), Glass Identification (glass), Landsat Satellite (satimage), and Yeast (yeast) with different class imbalance ratio as shown in Table 3. For highly imbalanced problems, the classes "headlamps", "damp grey soil", and "ME2" of glass, satimage, and yeast datasets, respectively, were converted into minority class and the remaining classes of each dataset became majority class. Except ionosphere, glass, and satimage, these datasets contain biomedical data.

The experiments were executed under the settings almost the same as above. The values for the performance criteria, sensitivity, specificity, and G-mean were calculated by averaging 20 independent runs of 10 -fold crossvalidation and summarized in Table 4 . The results also suggested that our method achieved better G-mean than the control method, SMOTE, safe-level-SMOTE, and borderline-SMOTE methods. Furthermore, the assessment by two-sample t-test showed that SMOTE, safelevel-SMOTE, and borderline-SMOTE significantly outperforms the control and our methods remarkably outperform the control, SMOTE, safe-level-SMOTE, and borderline-SMOTE with p-values smaller than 0.05 in most cases (for more details, see Table 5).

In addition, we calculated the correlation between the proportion of negative samples in the dataset (i.e. degree of imbalance) and improvement by the methods each with different classifiers as shown in Table 6. In case of SMOTE, the improvement from no-oversampling was calculated. However, in case of other methods, improvement from SMOTE was calculated. The results suggested that improvements by SMOTE, incrementalSMOTE2, and incremental-SMOTE3 have positive correlation to the degree of imbalance. In contrast, other methods have opposite characteristics. Among them, the improvements by SMOTE have shown relatively stronger correlation. These characteristics were observed more clearly when we used RF. Actually, only the combinations of RF with SMOTE, safelevel-SMOTE, and incremental-SMOTE2 showed the correlation values with $\mathrm{p}$ values less than 0.05 .

\section{CONCLUSION}

In this paper, we addressed a problem in human miRNA gene recognition, and showed that it requires a better 
Table 4. The comparison of Sensitivity (SE), Specificity (SP) and G-mean expressed in percent.

\begin{tabular}{|c|c|c|c|c|c|c|c|c|c|c|}
\hline \multirow{2}{*}{ Dataset } & \multirow{2}{*}{ Method } & \multicolumn{3}{|c|}{ SVM } & \multicolumn{3}{|c|}{ k-NN } & \multicolumn{3}{|c|}{ RF } \\
\hline & & SE & SP & G-mean & SE & SP & G-mean & SE & SP & G-mean \\
\hline \multirow{8}{*}{ Breast-w } & No over-sampling & 98.44 & 94.13 & 96.26 & 95.71 & 97.10 & 96.40 & 96.35 & 97.02 & 96.68 \\
\hline & SMOTE & 98.71 & 95.12 & 96.90 & 98.32 & 96.41 & 97.36 & 97.14 & 96.56 & 96.85 \\
\hline & Safelevel-SMOTE & 98.86 & 95.49 & 97.16 & 99.25 & 95.38 & 97.30 & 96.99 & 96.56 & 96.78 \\
\hline & Borderline-SMOTE1 & 99.07 & 94.00 & 96.50 & 98.40 & 95.82 & 97.10 & 96.78 & 96.66 & 96.72 \\
\hline & Borderline-SMOTE2 & 99.44 & 94.07 & 96.72 & 98.98 & 95.26 & 97.10 & 97.03 & 96.47 & 96.75 \\
\hline & increSMOTE1 & 99.44 & 95.48 & 97.44 & 99.25 & 96.05 & 97.64 & 97.43 & 96.56 & 96.99 \\
\hline & increSMOTE2 & 99.48 & 95.43 & 97.43 & 99.38 & 95.80 & 97.57 & 97.55 & 96.43 & 96.99 \\
\hline & increSMOTE3 & 99.54 & 95.41 & 97.46 & 99.23 & 95.90 & 97.55 & 97.51 & 96.48 & 97.00 \\
\hline \multirow{8}{*}{ Haberman } & No over-sampling & 18.77 & 92.87 & 41.71 & 28.09 & 87.87 & 49.65 & 23.52 & 91.13 & 46.26 \\
\hline & SMOTE & 53.21 & 65.49 & 58.99 & 55.43 & 65.38 & 60.15 & 41.48 & 77.04 & 56.49 \\
\hline & Safelevel-SMOTE & 66.79 & 56.18 & 61.22 & 66.05 & 54.36 & 59.89 & 44.57 & 75.71 & 58.06 \\
\hline & Borderline-SMOTE1 & 66.98 & 55.18 & 60.76 & 61.67 & 56.31 & 58.91 & 40.19 & 75.16 & 54.93 \\
\hline & Borderline-SMOTE2 & 69.07 & 54.67 & 61.41 & 69.14 & 49.73 & 58.61 & 41.17 & 74.13 & 55.20 \\
\hline & increSMOTE1 & 68.40 & 57.47 & 62.67 & 62.78 & 60.67 & 61.69 & 45.80 & 77.98 & 59.73 \\
\hline & increSMOTE2 & 68.02 & 57.36 & 62.44 & 63.83 & 59.96 & 61.81 & 50.37 & 76.00 & 61.84 \\
\hline & increSMOTE3 & 66.48 & 57.78 & 61.95 & 62.53 & 60.76 & 61.61 & 51.73 & 70.67 & 60.43 \\
\hline \multirow{8}{*}{ Blood } & No over-sampling & 30.65 & 94.21 & 53.71 & 27.56 & 91.03 & 50.07 & 29.24 & 90.04 & 51.27 \\
\hline & SMOTE & 74.04 & 60.35 & 66.84 & 51.88 & 74.70 & 62.24 & 50.28 & 72.47 & 60.35 \\
\hline & Safelevel-SMOTE & 65.76 & 68.61 & 67.08 & 57.67 & 68.39 & 62.79 & 54.07 & 71.11 & 62.00 \\
\hline & Borderline-SMOTE1 & 61.91 & 71.97 & 66.65 & 55.70 & 70.23 & 62.53 & 45.79 & 74.71 & 58.47 \\
\hline & Borderline-SMOTE2 & 50.11 & 84.89 & 65.22 & 63.96 & 62.87 & 63.39 & 55.31 & 64.75 & 59.81 \\
\hline & increSMOTE1 & 73.85 & 61.98 & 67.65 & 70.39 & 57.96 & 63.87 & 55.70 & 68.05 & 61.55 \\
\hline & increSMOTE2 & 73.17 & 62.71 & 67.74 & 58.03 & 69.37 & 63.43 & 63.96 & 63.42 & 63.68 \\
\hline & increSMOTE3 & 73.43 & 62.54 & 67.76 & 61.49 & 65.69 & 63.54 & 58.37 & 66.00 & 62.06 \\
\hline \multirow{8}{*}{ Breast-p } & No over-sampling & 10.11 & 99.47 & 31.36 & 20.85 & 87.88 & 42.69 & 19.68 & 98.54 & 43.87 \\
\hline & SMOTE & 53.30 & 73.81 & 62.68 & 59.47 & 57.58 & 58.45 & 43.30 & 83.68 & 60.16 \\
\hline & Safelevel-SMOTE & 52.98 & 74.93 & 62.98 & 61.17 & 56.62 & 58.80 & 41.91 & 84.54 & 59.45 \\
\hline & Borderline-SMOTE1 & 48.40 & 79.74 & 62.09 & 63.94 & 54.07 & 58.75 & 40.11 & 83.64 & 57.87 \\
\hline & Borderline-SMOTE2 & 50.64 & 73.64 & 61.00 & 68.40 & 50.63 & 58.81 & 50.43 & 70.13 & 59.38 \\
\hline & increSMOTE1 & 57.98 & 71.49 & 64.35 & 68.30 & 53.84 & 60.59 & 46.70 & 82.12 & 61.86 \\
\hline & increSMOTE2 & 59.89 & 69.27 & 64.39 & 68.72 & 53.44 & 60.58 & 48.72 & 81.66 & 63.05 \\
\hline & increSMOTE3 & 56.17 & 73.41 & 64.19 & 66.91 & 55.63 & 60.95 & 47.13 & 81.59 & 62.00 \\
\hline
\end{tabular}




\section{Continued}

\begin{tabular}{|c|c|c|c|c|c|c|c|c|c|c|}
\hline \multirow{8}{*}{ Yeast } & No over-sampling & 3.73 & 100.00 & 17.93 & 10.10 & 99.26 & 31.55 & 13.43 & 99.70 & 36.52 \\
\hline & SMOTE & 48.82 & 97.01 & 68.81 & 57.35 & 94.88 & 73.74 & 36.18 & 98.33 & 59.59 \\
\hline & Safelevel-SMOTE & 48.14 & 96.92 & 68.28 & 57.16 & 94.57 & 73.49 & 31.96 & 98.64 & 56.07 \\
\hline & Borderline-SMOTE1 & 42.45 & 97.60 & 64.31 & 47.94 & 94.96 & 67.42 & 24.41 & 99.06 & 49.10 \\
\hline & Borderline-SMOTE2 & 48.53 & 96.37 & 68.35 & 51.37 & 94.02 & 69.45 & 31.37 & 98.60 & 55.49 \\
\hline & increSMOTE1 & 50.59 & 96.82 & 69.97 & 59.22 & 94.61 & 74.81 & 38.14 & 98.30 & 61.16 \\
\hline & increSMOTE2 & 63.14 & 92.46 & 76.39 & 76.67 & 89.59 & 82.85 & 57.94 & 95.25 & 74.27 \\
\hline & increSMOTE3 & 61.27 & 91.98 & 75.04 & 71.47 & 91.28 & 80.73 & 48.04 & 96.77 & 68.12 \\
\hline \multirow{8}{*}{$\begin{array}{l}\text { Iono- } \\
\text { sphere }\end{array}$} & No over-sampling & 89.96 & 97.00 & 93.41 & 59.64 & 97.78 & 76.36 & 87.50 & 96.58 & 91.93 \\
\hline & SMOTE & 94.52 & 93.87 & 94.19 & 82.62 & 97.13 & 89.58 & 90.28 & 94.80 & 92.51 \\
\hline & Safelevel-SMOTE & 93.02 & 95.93 & 94.46 & 84.60 & 95.40 & 89.84 & 94.64 & 91.33 & 92.97 \\
\hline & Borderline-SMOTE1 & 92.34 & 96.64 & 94.47 & 82.54 & 95.80 & 88.91 & 91.23 & 93.82 & 92.51 \\
\hline & Borderline-SMOTE2 & 92.42 & 96.18 & 94.28 & 85.60 & 94.80 & 90.08 & 93.02 & 93.16 & 93.08 \\
\hline & increSMOTE1 & 93.61 & 96.24 & 94.92 & 86.39 & 96.16 & 91.14 & 94.52 & 93.18 & 93.84 \\
\hline & increSMOTE2 & 93.97 & 95.22 & 94.59 & 90.08 & 95.87 & 92.93 & 93.29 & 93.24 & 93.27 \\
\hline & increSMOTE3 & 93.89 & 95.33 & 94.61 & 91.19 & 93.36 & 92.26 & 92.74 & 94.02 & 93.38 \\
\hline \multirow{8}{*}{ Glass } & No over-sampling & 72.24 & 100.00 & 84.99 & 75.86 & 97.86 & 86.16 & 81.72 & 99.05 & 89.96 \\
\hline & SMOTE & 75.52 & 99.03 & 86.47 & 84.83 & 96.57 & 90.50 & 88.28 & 98.05 & 93.03 \\
\hline & Safelevel-SMOTE & 72.41 & 99.92 & 85.06 & 84.83 & 95.05 & 89.78 & 87.41 & 98.24 & 92.66 \\
\hline & Borderline-SMOTE1 & 72.76 & 99.62 & 85.13 & 83.79 & 96.78 & 90.04 & 87.93 & 98.16 & 92.90 \\
\hline & Borderline-SMOTE2 & 73.62 & 98.70 & 85.24 & 87.07 & 94.86 & 90.87 & 88.28 & 98.32 & 93.16 \\
\hline & increSMOTE1 & 76.72 & 99.38 & 87.31 & 87.24 & 96.35 & 91.68 & 89.31 & 98.41 & 93.75 \\
\hline & increSMOTE2 & 83.45 & 94.81 & 88.92 & 91.03 & 95.19 & 93.08 & 89.66 & 98.35 & 93.90 \\
\hline & increSMOTE3 & 76.03 & 99.78 & 87.10 & 91.03 & 95.14 & 93.05 & 89.66 & 98.38 & 93.92 \\
\hline \multirow{8}{*}{ Satimage } & No over-sampling & 51.26 & 97.99 & 70.88 & 67.16 & 97.15 & 80.78 & 52.88 & 98.94 & 72.33 \\
\hline & SMOTE & 85.30 & 92.62 & 88.88 & 89.83 & 91.01 & 90.42 & 68.20 & 96.85 & 81.27 \\
\hline & Safelevel-SMOTE & 86.53 & 92.20 & 89.32 & 91.65 & 89.32 & 90.48 & 67.71 & 97.09 & 81.08 \\
\hline & Borderline-SMOTE1 & 84.44 & 92.04 & 88.16 & 89.07 & 90.50 & 89.78 & 66.55 & 97.46 & 80.53 \\
\hline & Borderline-SMOTE2 & 87.71 & 91.13 & 89.40 & 91.08 & 89.14 & 90.10 & 69.67 & 96.95 & 82.18 \\
\hline & increSMOTE1 & 87.58 & 91.94 & 89.73 & 92.35 & 89.21 & 90.76 & 68.45 & 96.83 & 81.41 \\
\hline & increSMOTE2 & 90.51 & 91.88 & 91.19 & 93.33 & 88.20 & 90.73 & 76.49 & 94.70 & 85.11 \\
\hline & increSMOTE3 & 89.67 & 92.00 & 90.83 & 93.08 & 88.15 & 90.58 & 71.48 & 95.82 & 82.76 \\
\hline
\end{tabular}

method for the classification of human pre-miRNA hairpins from both pseudo hairpins and other ncRNAs; and it also was known as imbalanced class distribution problem. Then, we proposed a novel minority over-sampling method to deal with this imbalanced dataset problem. The novel method, incremental- SMOTE, was improved from SMOTE method, in which generated synthetic mi- nority class samples are utilized for further generation.

In order to compare the novel method with the control, SMOTE, and several successors of modified SMOTE, such as safe-level-SMOTE and borderline-SMOTE methods, we executed an experiment by 20 independent runs of 10 -fold cross-validation and t-test was also conducted to assess the statistical significance. The experi- 
Table 5. The assessment by two-sample t-test with equal variance.

\begin{tabular}{|c|c|c|c|c|c|c|}
\hline & & & & & $\mathrm{RF}$ & \\
\hline & & RF & SMOTE & $\mathrm{SL}^{\mathrm{a}}$ & $\mathrm{BL} 1^{\mathrm{b}}$ & $\mathrm{BL} 2^{\mathrm{c}}$ \\
\hline \multirow{7}{*}{ microPred } & SMOTE & $8.5 \mathrm{E}-09$ & $\mathrm{x}$ & $6.3 \mathrm{E}-01$ & $2.2 \mathrm{E}-02$ & $1.0 \mathrm{E}+00$ \\
\hline & safe-level & $7.9 \mathrm{E}-09$ & $3.6 \mathrm{E}-01$ & $\mathrm{x}$ & $1.1 \mathrm{E}-02$ & $1.0 \mathrm{E}+00$ \\
\hline & borderline1 & $4.2 \mathrm{E}-07$ & $9.7 \mathrm{E}-01$ & & $\mathrm{x}$ & $1.0 \mathrm{E}+00$ \\
\hline & borderline 2 & $2.2 \mathrm{E}-16$ & $1.6 \mathrm{E}-09$ & $1.9 \mathrm{E}-08$ & $7.5 \mathrm{E}-15$ & $\mathrm{x}$ \\
\hline & increSMOTE1 & $9.8 \mathrm{E}-13$ & $1.0 \mathrm{E}-02$ & $3.0 \mathrm{E}-02$ & $9.1 \mathrm{E}-06$ & $1.0 \mathrm{E}+00$ \\
\hline & increSMOTE2 & $4.9 \mathrm{E}-14$ & $3.0 \mathrm{E}-03$ & $1.1 \mathrm{E}-02$ & $5.3 \mathrm{E}-07$ & $1.0 \mathrm{E}+00$ \\
\hline & increSMOTE3 & $2.6 \mathrm{E}-14$ & $3.0 \mathrm{E}-03$ & $1.1 \mathrm{E}-02$ & $2.7 \mathrm{E}-07$ & $1.0 \mathrm{E}+00$ \\
\hline \multirow{7}{*}{ miRNA-network } & SMOTE & $2.2 \mathrm{E}-16$ & $\mathrm{x}$ & $1.7 \mathrm{E}-01$ & $1.9 \mathrm{E}-03$ & $4.6 \mathrm{E}-04$ \\
\hline & safe-level & $2.2 \mathrm{E}-16$ & $8.2 \mathrm{E}-01$ & $\mathrm{x}$ & $2.0 \mathrm{E}-02$ & $3.6 \mathrm{E}-04$ \\
\hline & borderline1 & $2.2 \mathrm{E}-16$ & $9.9 \mathrm{E}-01$ & & $\mathrm{x}$ & $1.2 \mathrm{E}-01$ \\
\hline & borderline2 & $3.8 \mathrm{E}-12$ & $9.9 \mathrm{E}-01$ & & & $\mathrm{x}$ \\
\hline & increSMOTE1 & $2.2 \mathrm{E}-16$ & $2.6 \mathrm{E}-02$ & $6.2 \mathrm{E}-03$ & $2.7 \mathrm{E}-05$ & $1.8 \mathrm{E}-05$ \\
\hline & increSMOTE2 & $2.2 \mathrm{E}-16$ & $4.0 \mathrm{E}-01$ & $1.5 \mathrm{E}-01$ & $2.3 \mathrm{E}-03$ & $4.5 \mathrm{E}-04$ \\
\hline & increSMOTE3 & $2.2 \mathrm{E}-16$ & $5.1 \mathrm{E}-01$ & $2.0 \mathrm{E}-01$ & $3.3 \mathrm{E}-03$ & $6.4 \mathrm{E}-04$ \\
\hline \multirow{7}{*}{ Breast-w } & SMOTE & $5.1 \mathrm{E}-02$ & $\mathrm{x}$ & $2.2 \mathrm{E}-01$ & $9.0 \mathrm{E}-02$ & $1.6 \mathrm{E}-01$ \\
\hline & Safe-level & $1.9 \mathrm{E}-01$ & $7.7 \mathrm{E}-01$ & $\mathrm{x}$ & $2.9 \mathrm{E}-01$ & $4.1 \mathrm{E}-01$ \\
\hline & borderline1 & $3.6 \mathrm{E}-01$ & $9.0 \mathrm{E}-01$ & & $\mathrm{x}$ & $6.1 \mathrm{E}-01$ \\
\hline & borderline2 & $2.6 \mathrm{E}-01$ & $8.3 \mathrm{E}-01$ & & & $\mathrm{x}$ \\
\hline & increSMOTE1 & $1.3 \mathrm{E}-03$ & $4.0 \mathrm{E}-02$ & $1.0 \mathrm{E}-02$ & $3.0 \mathrm{E}-03$ & $7.0 \mathrm{E}-03$ \\
\hline & increSMOTE2 & $1.5 \mathrm{E}-03$ & $4.0 \mathrm{E}-02$ & $1.0 \mathrm{E}-02$ & $3.0 \mathrm{E}-03$ & $8.0 \mathrm{E}-03$ \\
\hline & increSMOTE3 & $2.2 \mathrm{E}-03$ & $5.8 \mathrm{E}-02$ & $1.0 \mathrm{E}-02$ & $5.0 \mathrm{E}-03$ & $1.0 \mathrm{E}-02$ \\
\hline \multirow{7}{*}{ Haberman } & SMOTE & $2.2 \mathrm{E}-16$ & $\mathrm{x}$ & $9.9 \mathrm{E}-01$ & $9.1 \mathrm{E}-03$ & $3.0 \mathrm{E}-02$ \\
\hline & safe-level & $2.2 \mathrm{E}-16$ & $6.0 \mathrm{E}-03$ & $\mathrm{x}$ & $4.2 \mathrm{E}-08$ & $5.3 \mathrm{E}-06$ \\
\hline & borderline 1 & $2.2 \mathrm{E}-16$ & $9.9 \mathrm{E}-01$ & & $\mathrm{x}$ & $6.7 \mathrm{E}-01$ \\
\hline & borderline 2 & $2.2 \mathrm{E}-16$ & $9.6 \mathrm{E}-01$ & & & $\mathrm{x}$ \\
\hline & increSMOTE1 & $2.2 \mathrm{E}-16$ & $2.4 \mathrm{E}-05$ & $3.1 \mathrm{E}-03$ & $1.2 \mathrm{E}-09$ & $2.5 \mathrm{E}-08$ \\
\hline & increSMOTE2 & $2.2 \mathrm{E}-16$ & $3.1 \mathrm{E}-10$ & $8.5 \mathrm{E}-10$ & $2.8 \mathrm{E}-16$ & $1.1 \mathrm{E}-13$ \\
\hline & increSMOTE3 & $2.2 \mathrm{E}-16$ & $7.5 \mathrm{E}-07$ & $7.1 \mathrm{E}-05$ & $1.6 \mathrm{E}-11$ & $5.4 \mathrm{E}-10$ \\
\hline \multirow{7}{*}{ Blood } & SMOTE & $2.2 \mathrm{E}-16$ & $\mathrm{x}$ & & $6.7 \mathrm{E}-08$ & $7.1 \mathrm{E}-02$ \\
\hline & Safe-level & $2.2 \mathrm{E}-16$ & $1.1 \mathrm{E}-05$ & $\mathrm{x}$ & $2.7 \mathrm{E}-12$ & $2.3 \mathrm{E}-06$ \\
\hline & borderline1 & $2.6 \mathrm{E}-14$ & $1.0 \mathrm{E}+00$ & & $\mathrm{x}$ & $9.9 \mathrm{E}-01$ \\
\hline & borderline 2 & $2.2 \mathrm{E}-16$ & $9.2 \mathrm{E}-01$ & & $4.0 \mathrm{E}-04$ & $\mathrm{x}$ \\
\hline & increSMOTE1 & $2.2 \mathrm{E}-16$ & $1.0 \mathrm{E}-03$ & $8.5 \mathrm{E}-01$ & $1.3 \mathrm{E}-09$ & $1.5 \mathrm{E}-04$ \\
\hline & increSMOTE2 & $2.2 \mathrm{E}-16$ & $1.5 \mathrm{E}-12$ & $3.5 \mathrm{E}-05$ & $2.2 \mathrm{E}-16$ & $4.6 \mathrm{E}-12$ \\
\hline & increSMOTE3 & $2.2 \mathrm{E}-16$ & $1.5 \mathrm{E}-06$ & $4.3 \mathrm{E}-01$ & $1.3 \mathrm{E}-13$ & $6.5 \mathrm{E}-07$ \\
\hline
\end{tabular}




\section{Continued}

Breast-p

Yeast

SMOTE $2.6 \mathrm{E}-16 \quad x$

\begin{tabular}{|c|c|c|c|c|c|c|}
\hline & Safe-level & $2.2 \mathrm{E}-16$ & $8.2 \mathrm{E}-01$ & $\mathrm{x}$ & $3.0 \mathrm{E}-02$ & $4.6 \mathrm{E}-01$ \\
\hline & borderline1 & $2.6 \mathrm{E}-15$ & $9.9 \mathrm{E}-01$ & & $\mathrm{x}$ & $9.6 \mathrm{E}-01$ \\
\hline \multirow[t]{7}{*}{ Breast-p } & borderline2 & $2.2 \mathrm{E}-16$ & $8.5 \mathrm{E}-01$ & & $3.0 \mathrm{E}-02$ & $\mathrm{x}$ \\
\hline & increSMOTE1 & $2.2 \mathrm{E}-16$ & $1.0 \mathrm{E}-02$ & $5.0 \mathrm{E}-03$ & $1.4 \mathrm{E}-05$ & $3.0 \mathrm{E}-03$ \\
\hline & increSMOTE2 & $2.2 \mathrm{E}-16$ & $2.8 \mathrm{E}-05$ & $3.9 \mathrm{E}-05$ & $1.4 \mathrm{E}-08$ & $2.1 \mathrm{E}-05$ \\
\hline & increSMOTE3 & $2.2 \mathrm{E}-16$ & $2.0 \mathrm{E}-03$ & $1.0 \mathrm{E}-03$ & $6.9 \mathrm{E}-07$ & $7.0 \mathrm{E}-04$ \\
\hline & SMOTE & $2.2 \mathrm{E}-16$ & $\mathrm{x}$ & $1.5 \mathrm{E}-04$ & $1.5 \mathrm{E}-15$ & $1.4 \mathrm{E}-04$ \\
\hline & Safe-level & $2.2 \mathrm{E}-16$ & $9.9 \mathrm{E}-01$ & $\mathrm{x}$ & $2.5 \mathrm{E}-09$ & $3.0 \mathrm{E}-01$ \\
\hline & borderline1 & $2.2 \mathrm{E}-16$ & $1.0 \mathrm{E}+00$ & & $\mathrm{x}$ & $1.0 \mathrm{E}+00$ \\
\hline \multirow[t]{7}{*}{ Yeast } & borderline 2 & $2.2 \mathrm{E}-16$ & $9.9 \mathrm{E}-01$ & & & $\mathrm{x}$ \\
\hline & increSMOTE1 & $2.2 \mathrm{E}-16$ & $3.0 \mathrm{E}-02$ & $1.9 \mathrm{E}-06$ & $2.2 \mathrm{E}-16$ & $2.8 \mathrm{E}-06$ \\
\hline & increSMOTE2 & $2.2 \mathrm{E}-16$ & $2.2 \mathrm{E}-16$ & $2.2 \mathrm{E}-16$ & $2.2 \mathrm{E}-16$ & $2.2 \mathrm{E}-16$ \\
\hline & increSMOTE3 & $2.2 \mathrm{E}-16$ & $6.2 \mathrm{E}-12$ & $3.2 \mathrm{E}-15$ & $2.2 \mathrm{E}-16$ & $4.3 \mathrm{E}-14$ \\
\hline & SMOTE & $1.3 \mathrm{E}-04$ & $\mathrm{x}$ & & & \\
\hline & Safe-level & $3.4 \mathrm{E}-05$ & $2.0 \mathrm{E}-02$ & $\mathrm{x}$ & $4.0 \mathrm{E}-02$ & $6.5 \mathrm{E}-01$ \\
\hline & borderline1 & $9.0 \mathrm{E}-04$ & $4.0 \mathrm{E}-01$ & & $\mathrm{x}$ & $9.8 \mathrm{E}-01$ \\
\hline \multirow[t]{7}{*}{ Ionosphere } & borderline2 & $3.6 \mathrm{E}-06$ & $7.0 \mathrm{E}-03$ & & & $\mathrm{x}$ \\
\hline & increSMOTE1 & $1.6 \mathrm{E}-13$ & $4.1 \mathrm{E}-09$ & $5.5 \mathrm{E}-04$ & $5.9 \mathrm{E}-08$ & $1.2 \mathrm{E}-03$ \\
\hline & increSMOTE2 & $1.2 \mathrm{E}-09$ & $9.3 \mathrm{E}-05$ & $1.1 \mathrm{E}-01$ & $3.0 \mathrm{E}-04$ & $2.2 \mathrm{E}-01$ \\
\hline & increSMOTE3 & $2.5 \mathrm{E}-07$ & $3.9 \mathrm{E}-04$ & $8.1 \mathrm{E}-02$ & $7.0 \mathrm{E}-04$ & $1.4 \mathrm{E}-01$ \\
\hline & SMOTE & $7.1 \mathrm{E}-08$ & $\mathrm{x}$ & $1.5 \mathrm{E}-01$ & $3.7 \mathrm{E}-01$ & $6.2 \mathrm{E}-01$ \\
\hline & Safe-level & $6.1 \mathrm{E}-07$ & $8.4 \mathrm{E}-01$ & $\mathrm{x}$ & $7.1 \mathrm{E}-01$ & $9.0 \mathrm{E}-01$ \\
\hline & borderline1 & $3.9 \mathrm{E}-07$ & $6.2 \mathrm{E}-01$ & & $\mathrm{x}$ & $7.3 \mathrm{E}-01$ \\
\hline \multirow[t]{7}{*}{ Glass } & borderline2 & $4.1 \mathrm{E}-08$ & $3.7 \mathrm{E}-01$ & & & $\mathrm{x}$ \\
\hline & increSMOTE1 & $1.1 \mathrm{E}-09$ & $1.1 \mathrm{E}-02$ & $2.9 \mathrm{E}-04$ & $9.6 \mathrm{E}-03$ & $3.0 \mathrm{E}-02$ \\
\hline & increSMOTE2 & $1.4 \mathrm{E}-09$ & $1.7 \mathrm{E}-03$ & $3.5 \mathrm{E}-05$ & $2.2 \mathrm{E}-03$ & $7.0 \mathrm{E}-03$ \\
\hline & increSMOTE3 & $1.3 \mathrm{E}-09$ & $1.5 \mathrm{E}-03$ & $3.1 \mathrm{E}-05$ & $2.0 \mathrm{E}-03$ & $6.0 \mathrm{E}-03$ \\
\hline & SMOTE & $2.2 \mathrm{E}-16$ & $\mathrm{x}$ & $6.4 \mathrm{E}-02$ & $7.1 \mathrm{E}-08$ & $1.0 \mathrm{E}+00$ \\
\hline & Safe-level & $2.2 \mathrm{E}-16$ & $9.3 \mathrm{E}-01$ & $\mathrm{x}$ & $3.0 \mathrm{E}-05$ & $1.0 \mathrm{E}+00$ \\
\hline & borderline1 & $2.2 \mathrm{E}-16$ & $1.0 \mathrm{E}+00$ & & $\mathrm{x}$ & $1.0 \mathrm{E}+00$ \\
\hline \multirow[t]{4}{*}{ Satimage } & borderline2 & $2.2 \mathrm{E}-16$ & $3.2 \mathrm{E}-08$ & $7.6 \mathrm{E}-10$ & $5.7 \mathrm{E}-15$ & $\mathrm{x}$ \\
\hline & increSMOTE1 & $2.2 \mathrm{E}-16$ & $1.0 \mathrm{E}-01$ & $2.7 \mathrm{E}-03$ & $6.9 \mathrm{E}-11$ & $1.0 \mathrm{E}+00$ \\
\hline & increSMOTE2 & $2.2 \mathrm{E}-16$ & $2.2 \mathrm{E}-16$ & $2.2 \mathrm{E}-16$ & $2.2 \mathrm{E}-16$ & $2.2 \mathrm{E}-16$ \\
\hline & increSMOTE3 & $2.2 \mathrm{E}-16$ & $3.3 \mathrm{E}-16$ & $2.2 \mathrm{E}-16$ & $2.2 \mathrm{E}-16$ & $2.2 \mathrm{E}-05$ \\
\hline
\end{tabular}


Table 6. The correlation between degree of imbalance and improvement by the methods each with different classifiers.

\begin{tabular}{lccc}
\hline & SVM & k-NN & RF \\
\hline SMOTE & 0.70 & 0.42 & 0.94 \\
Safelevel-SMOTE & -0.59 & -0.31 & -0.72 \\
Borderline-SMOTE1 & -0.54 & -0.49 & -0.65 \\
Borderline-SMOTE2 & 0.01 & -0.39 & -0.35 \\
increSMOTE1 & -0.66 & -0.50 & -0.48 \\
increSMOTE2 & 0.66 & 0.23 & 0.83 \\
increSMOTE3 & 0.36 & 0.21 & 0.59 \\
\hline
\end{tabular}

mental results showed that our method achieved better G-mean and Sensitivity than both of the control, SMOTE, safe-level-SMOTE, and borderline-SMOTE methods with the p-value less than 0.05 in most cases. These results suggest that our method outperforms SMOTE and several successors of modified SMOTE in various biomedical classification problems, including human miRNA gene prediction.

Although incremental-SMOTE achieved better performances in various biomedical classification problems, the advantages and disadvantages of three variations of incremental-SMOTE are still unclear in real applications. Moreover, there are still several topics left to be considered further such as: the combination of our novel method with feature selection methods, application of other novel under-sampling methods, extraction of a new and appropriate set of features from pre-miRNA hairpins dataset, and so on. In future work, we will find the solution to these problems.

\section{ACKNOWLEDGEMENTS}

The authors wish to thank Batuwita et al. and Xiao et al. for providing us with microPred and miRNA network-level datasets, respectively.

\section{REFERENCES}

[1] Kim, V.N. and Nam, J.-W. (2006) Genomics of microRNA. Trends in Genetic, 22, 165-173. doi:10.1016/j.tig.2006.01.003

[2] Lee, R.C., Feinbaum, R.L. and Ambros, V. (1993) The C. elegans heterochronic gene lin-4 encodes small RNAs with antisense complementarity to lin-14. Cell, 75, 843854. doi:10.1016/0092-8674(93)90529-Y

[3] Harfe, B.D., Mcmanus, M.T., Mansfield, J.H., Hornstein, E. and Tabin, C.J. (2005) The RNaseIII enzyme Dicer is required for morphogenesis but not patterning of the vertebrate limb. Proceedings of the National Academy of Sciences, 102, 10898-10903. doi: $10.1073 /$ pnas. 0504834102

[4] Wilfred, B.R., Wang, W. and Nelson, P.T. (2007) Ener- gizing miRNA research: A review of the role of miRNAs in lipid metabolism, with a prediction that miR-103/107 regulates human metabolic pathways. Molecular Genetics and Metabolism, 91:209-217. doi:10.1016/j.ymgme.2007.03.011

[5] Lodish, H.F., Chen, C. and Bartel, D.P. (2004) MicroRNAs modulate hematopoietic lineage differentiation. Science, 303, 83-86. doi:10.1126/science.1091903

[6] Lim, L.P., Lau, N.C., Garrett-engele, P. and Grimson, A. (2005) Microarray analysis shows that some microRNAs downregulate large numbers of target mRNAs. Nature, 292, 288-292.

[7] Kozomara, A. and Griffiths-Jones, S. (2011) miRBase: Integrating microRNA annotation and deep-sequencing data. Nucleic Acids Research, 39, D152-D157. doi:10.1093/nar/gkq1027

[8] Hertel, J. and Stadler, P.F. (2006) Hairpins in a Haystack: Recognizing microRNA precursors in comparative genomics data. Bioinformatics, 22, e197-e202. doi:10.1093/bioinformatics/btl257

[9] Lim, L.P., Glasner, M.E., Yekta, S., Burge, C.B. and Bartel, D.P. (2003) Vertebrate microRNA genes. Science, 299, 1540. doi:10.1126/science. 1080372

[10] Lai, E.C., Tomancak, P., Williams, R.W. and Rubin, G.M. (2003) Computational identification of Drosophila microRNA genes. Genome Biology, 4, R42. doi:10.1186/gb-2003-4-7-r42

[11] Jones-Rhoades, M.W. and Bartel, D.P. (2004) Computational identification of plant microRNAs and their targets, including a stress-induced miRNA. Molecular Cell, 14, 787-799. doi:10.1016/j.molcel.2004.05.027

[12] Bonnet, E., Wuyts, J., Rouzé, P. and Van de Peer, Y. (2004) Detection of 91 potential conserved plant microRNAs in Arabidopsis thaliana and Oryza sativa identifies important target genes. Proceedings of the National Academy of Sciences, 101, 11511-11516. doi:10.1073/pnas.0404025101

[13] Ng, K.L.S. and Mishra, S.K. (2007) De novo SVM classification of precursor microRNAs from genomic pseudo hairpins using global and intrinsic folding measures. Bioinformatics, 23, 1321-1330. doi:10.1093/bioinformatics/btm026

[14] Berezikov, E., Guryev, V., Van de Belt, J., Wienholds, E., Plasterk, R.H.A. and Cuppen, E. (2005) Phylogenetic shadowing and computational identification of human microRNA genes. Cell, 120, 21-24. doi:10.1016/j.cell.2004.12.031

[15] Sewer, A., Paul, N., Landgraf, P., Aravin, A., Pfeffer, S., Brownstein, M.J., Tuschl, T., Van Nimwegen, E. and Zavolan, M. (2005) Identification of clustered microRNAs using an $a b$ initio prediction method. BMC Bioinformatics, 6, 267. doi:10.1186/1471-2105-6-267

[16] Xue, C., Li, F., He, T., Liu, G-.P., Li, Y. and Zhang, X. (2005) Classification of real and pseudo microRNA precursors using local structure-sequence features and support vector machine. BMC Bioinformatics, 6, 310 . doi:10.1186/1471-2105-6-310

[17] Clote, P., Ferré, F., Kranakis, E. and Krizanc, D. (2005) 
Structural RNA has lower folding energy than random RNA of the same dinucleotide frequency. RNA, 11, 578591. doi:10.1261/rna.7220505

[18] Jiang, P., Wu, H., Wang, W., Ma, W., Sun, X. and Lu, Z. (2007) MiPred: Classification of real and pseudo microRNA precursors using random forest prediction model with combined features. Nucleic Acids Research, 35, 339344. doi:10.1093/nar/gkm368

[19] Tang, X., Xiao, J., Li, Y., Wen, Z., Fang, Z. and Li, M. (2012) Systematic analysis revealed better performance of random forest algorithm coupled with complex network features in predicting microRNA precursors. Chemometrics and Intelligent Laboratory Systems, 118, $317-$ 323. doi:10.1016/j.chemolab.2012.05.001

[20] Wang, Y., Chen, X., Jiang, W., Li, L., Li, W., Yang, L., Liao, M., Lian, B., Lv, Y., Wang, S., Wang, S. and Li, X. (2011) Predicting human microRNA precursors based on an optimized feature subset generated by GA-SVM. Genomics, 98, 73-78. doi:10.1016/j.ygeno.2011.04.011

[21] Zhang, Y., Yang, Y., Zhang, H., Jiang, X., Xu, B., Xue, Y., Cao, Y., Zhai, Q., Zhai, Y., Xu, M., Cooke, H.J. and Shi, Q. (2011) Prediction of novel pre-microRNAs with high accuracy through boosting and SVM. Bioinformatics, 27, 1436-1437. doi:10.1093/bioinformatics/btr148

[22] Kadri, S., Hinman, V. and Benos, P.V. (2009) HHMMiR: Efficient de novo prediction of microRNAs using hierarchical hidden Markov models. BMC Bioinformatics, 10, S35. doi:10.1186/1471-2105-10-S1-S35

[23] Batuwita, R. and Palade, V. (2009) microPred: Effective classification of pre-miRNAs for human miRNA gene prediction. Bioinformatics, 25, 989-995. doi:10.1093/bioinformatics/btp107

[24] Xiao, J., Tang, X., Li, Y., Fang, Z., Ma, D., He, Y. and Li, M. (2011) Identification of microRNA precursors based on random forest with network-level representation method of stem-loop structure. BMC Bioinformatics, 12, 165. doi:10.1186/1471-2105-12-165

[25] Bunkhumpornpat, C., Sinapiromsaran, K. and Lursinsap, C. (2009) Safe-Level-SMOTE: Safe-level-synthetic minority over-sampling technique. Lecture Notes in Computer Science, 5476, 475-482. doi:10.1007/978-3-642-01307-2_43

[26] Han, H., Wang, W. and Mao, B. (2005) Borderline-
SMOTE: A new over-sampling method in imbalanced data sets learning. Lecture Notes in Computer Science, 3644, 878-887. doi:10.1007/11538059 91

[27] Chawla, N.V., Bowyer, K.W. and Hall, L.O. (2002) SMOTE: Synthetic minority over-sampling technique. Journal of Artificial Intelligence Research, 16, 321-357.

[28] Burges, C.J.C. (1998) A tutorial on support vector machines for pattern recognition. Data Mining and Knowledge Discovery, 2, 121-167. doi:10.1023/A:1009715923555

[29] Vapnik, V.N. (1999) An overview of statistical learning theory. IEEE Transactions on Neural networks, 10, 988999. doi: $10.1109 / 72.788640$

[30] Karatzoglou, A. and Smola, A. (2004) kernlab-An S4 package for kernel methods in R. Journal of Statistical Software, 11, 1-20.

[31] Venables, W.N. and Ripley, B.D. (2002) Modern applied statistics with S. 4th Edition. Springer, New York. doi:10.1007/978-0-387-21706-2

[32] Liaw, A. and Wiener, M. (2002) Classification and regression by random forest. $R$ News, 2, 18-22.

[33] Akbani, R., Kwek, S. and Japkowicz, N. (2004) Applying support vector machines to imbalanced datasets. Lecture Notes in Computer Science, 3201, 39-50. doi:10.1007/978-3-540-30115-8 7

[34] Anand, A., Pugalenthi, G., Fogel, G.B. and Suganthan, P.N. (2010) An approach for classification of highly imbalanced data using weighting and undersampling. Amino Acids, 39, 1385-1391. doi:10.1007/s00726-010-0595-2

[35] Kubat, M. and Matwin, S. (8-12 July 1997) Addressing the curse of imbalanced training sets: One-sided selection. Proceedings of the 14th International Conference in Machine Learning, Nashville, 179-186.

[36] Han, K. (2011) Effective sample selection for classification of pre-miRNAs. Genetics and Molecular Research, 10, 506-518. doi:10.4238/vol10-1gmr1054

[37] Xuan, P., Guo, M., Liu, X., Huang, Y., Li, W. and Huang, Y. (2011) PlantMiRNAPred: Efficient classification of real and pseudo plant pre-miRNAs. Bioinformatics, 27, 1368-1376. doi:10.1093/bioinformatics/btr153

[38] Frank, A. and Asuncion, A. (2010) UCI machine learning repository. University of California, Irvine. 